\title{
Neuronal and endothelial nitric oxide synthase gene knockout mice
}

\section{P.L. Huang}

\section{Abstract}

\section{Correspondence}

P.L. Huang

Cardiovascular Research Center

Massachusetts General Hospital East

149 East 13th Street

Charlestown, MA 02129

USA

Fax: + 1-617-726-5806

E-mail:

huangP@ helix.mgh.harvard.edu

Presented at the Meeting

"NO Brazil, Basic and Clinical

Aspects of Nitric $O$ xide",

Foz do Iguaçu, PR, Brazil,

March 10-13, 1999.

Research supported by the National Institute of Neurologic Diseases and Stroke (No. NS33335), the National Heart, Lung and Blood Institute (No. HL 52818), and an Established Investigator Award from the American Heart Association.

Received May 28, 1999

Accepted June 22, 1999

\author{
Department of Medicine, Harvard Medical School, \\ Cardiovascular Research Center and Cardiology Division \\ Massachusetts General Hospital, Boston, MA, USA
}

Targeted disruption of the neuronal nitric oxide synthase (nNOS) and endothelial nitric oxide synthase (eNOS) genes has led to knockout mice that lack these isoforms. These animal models have been useful to study the roles of nitric oxide (NO) in physiologic processes. nNOS knockout mice have enlarged stomachs and defects in the inhibitory junction potential involved in gastrointestinal motility. eNOS knockout mice are hypertensive and lack endothelium-derived relaxing factor activity. When these animals are subjected to models of focal ischemia, the nNOS mutant mice develop smaller infarcts, consistent with a role for nNOS in neurotoxicity following cerebral ischemia. In contrast, eNOS mutant mice develop larger infarcts, and show a more pronounced hemodynamic effect of vascular occlusion. The knockout mice also show that $\mathrm{nNOS}$ and $\mathrm{eNOS}$ isoforms differentially modulate the release of neurotransmitters in various regions of the brain. eNOS knockout mice respond to vessel injury with greater neointimal proliferation, confirming that reduced NO levels seen in endothelial dysfunction change the vessel response to injury. Furthermore, eNOS mutant mice still show a protective effect of female gender, indicating that the mechanism of this protection cannot be limited to upregulation of eNOS expression. The eNOS mutant mice also prove that eNOS modulates the cardiac contractile response to $ß$-adrenergic agonists and baseline diastolic relaxation. Atrial natriuretic peptide, upregulated in the hearts of eNOS mutant mice, normalizes cGMP levels and restores normal diastolic relaxation.

\section{Introduction}

Nitric oxide (NO) is a ubiquitous molecule that serves many biological functions (1). It is an important endogenous vasodilator responsible for endothelium-derived relaxing factor (EDRF) activity. It also serves other important functions in the vasculature, including suppression of smooth muscle cell proliferation, modulation of expression of
Key words

- Nitric oxide

- Targeted disruption

- Gene knockout

- Endothelium

- Stroke

- Atherosclerosis adhesion molecules, and modulation of leukocyte activation, leukocyte adhesion, and platelet aggregation. In the peripheral nervous system, NO serves as a neurotransmitter in nonadrenergic, noncholinergic nerves in the gastrointestinal, respiratory, and genitourinary tracts. In the central nervous system, NO is an important link between glutamate activation of NMDA receptors and stimulation of guanylyl cyclase. NO is also gen- 
erated in large quantities by cells of macrophage lineage as a defense mechanism against pathogens and tumor cells.

As a gas, NO diffuses freely across cell membranes, so it does not require specialized apparatus for its storage, secretion, or binding. It has a short biological half-life, since it is rapidly inactivated by circulating hemoglobin and reacts rapidly with superoxide to form peroxynitrite anion. Many biological effects of NO are attributable to the activation of guanylyl cyclase when NO binds to its heme group. However, NO has additional mechanisms of action that are independent of cGMP, including inhibition of mitochondrial complexes I and IV, activation of potassium-dependent calcium channels, formation of peroxynitrite anion, and binding to iron or sulfur proteins.

NO is generated by the nitric oxide synthase (NOS) enzymes, which use L-arginine as substrate (2). There are at least three major NOS isoforms, each encoded by separate genes: neuronal NOS (nNOS, type I NOS), inducible NOS (iNOS, type II NOS, also macrophage NOS), and endothelial NOS (eNOS, type III NOS). These isoforms were named for the tissues in which they were first discovered, but their expression patterns are now known to overlap in a complex manner. For example, muscle cells are known to express all three NOS isoforms. Both nNOS and eNOS are expressed in neurons in the brain. Vascular endothelial cells express eNOS but can be induced to express iNOS.

All NOS isoforms share identical substrates and possess similarities in enzyme mechanism, so they also share overlapping sensitivities to pharmacologic inhibitors. This is especially true for arginine analogs such as L-N-arginine methyl ester (L-NAME), L$\mathrm{N}$-monomethylarginine (L-NMMA), and Lnitroarginine (L-NA). Some inhibitors demonstrate relative selectivity between NOS isoforms. For example, 7-nitroindazole is relatively specific for nNOS in vivo. Aminoguanidine is relatively specific for iNOS.
However, lack of specificity is a major limitation of NOS inhibitors in studies of specific roles of individual NOS isoforms.

A separate approach to the study of individual NOS isoforms is targeted disruption of the genes for NOS isoforms, creating gene knockout mice. This provides genetic specificity of deletion of individual NOS isoforms. Each of the NOS isoforms has been knocked out, leading to viable and fertile animals that serve as useful tools to study the roles of the deleted genes.

\section{Generation and characterization of nNOS knockout mice}

The nNOS gene was disrupted by standard techniques of homologous recombination in embryonic stem cells, deleting the first protein coding exon of nNOS, including the translation initiation codon ATG (3). This results in the elimination of $95-99 \%$ of nNOS expression, leaving only variant transcripts that lack the first exon and that are present at extremely low levels. The deleted segment also contains the PDZ domains of the nNOS protein that are critical for the association of the protein to membranes (4).

Homozygous nNOS knockout mice are viable and fertile. Immunostaining for $\mathrm{nNOS}$ and NADPH diaphorase staining, which stains for nNOS enzymatic activity on nitroblue tetrazolium substrate, are absent in the brain and in peripheral tissues, while they are clearly noted in wild-type mice. The most apparent phenotype of the nNOS knockout mice is enlargement of the stomachs and pyloric stenosis (3). Staining of stomach muscle demonstrates absence of the normal staining for nNOS or NADPH diaphorase in the myenteric nerve plexus. Both enlargement of the stomach and lack of NADPH diaphorase staining are noted in children with infantile hypertrophic pyloric stenosis, consistent with a physiologic requirement for nNOS in gastrointestinal motility. Stomach muscle strips from nNOS knockout mice 
also display altered inhibitory junction potentials (5).

\section{Generation and characterization of eNOS knockout mice}

Regions that encode the NADPH ribose and adenine-binding sites were disrupted to create the eNOS gene knockout mice. The mutant mice are viable and fertile, and show no gross anatomic abnormalities (6). However, enzymatic assay confirms lack of eNOS activity, and immunohistochemistry shows lack of eNOS expression in all tissues examined. Isolated strips of aorta show complete absence of EDRF activity, as defined by dose-dependent acetylcholine-induced vascular relaxation, while strips from wild-type mice show EDRF activity that can be blocked by NOS inhibitors. The blood pressure of the eNOS knockout mice is consistently $20-30 \%$ higher than that of wild-type animals, compared under identical conditions of anesthesia and in the awake state (6). Thus, hypertension results from the absence of this endogenous vasodilator. This may be caused both by the requirement for eNOS-derived NO for vascular tone and by the involvement of eNOS in determining the baroreceptor blood pressure setpoint.

\section{Neuronal NOS contributes to toxicity following cerebral ischemia}

Levels of NO rise from nanomolar concentrations to micromolar concentrations following cerebral ischemia (7). There is also a corresponding increase in brain cGMP levels following ischemia. These effects are mediated by nNOS, since they are absent in the nNOS mutant mice. Basal levels of NO and cGMP in the brain are lower in the nNOS knockout animals and do not increase following ischemia (8).

In order to determine the effect of nNOSderived NO on cerebral ischemia, we subjected nNOS knockout mice and wild-type mice to models of cerebral ischemia. When subjected to a middle cerebral artery occlusion (MCAO) model of focal ischemia, the nNOS knockout mice developed substantially smaller infarcts than did the wild-type mice (8). Examination of the vessel calibers and arrangement in the circle of Willis showed no differences between the two groups. Measurement of blood flow reductions by laser Doppler flowmetry showed comparable reductions in cerebral blood flow, both in the core ischemic area and the surrounding ischemia penumbra. Thus, vascular anatomic or functional differences do not account for the protection seen in the nNOS knockout mice. These results indicate that nNOS contributes to the toxicity seen following cerebral ischemia. Protection was noted in the nNOS knockouts for both transient and permanent focal ischemia $(8,9)$, and in models of global ischemia (10).

There are several mechanisms by which nNOS may contribute to neuronal toxicity. NO reacts with superoxide to form peroxynitrite, which itself is toxic and can nitrosylate tyrosine residues in proteins (11). Nitrosotyrosine is a marker for peroxynitrite formation and can be detected immunohistochemically. Nitrosylated tyrosine residues may not function properly if phosphorylation of those residues is important to normal physiology. Nitrotyrosine formation is dramatically reduced in nNOS knockout mice following cerebral ischemia (12). NO may also activate poly-ADP ribose polymerase (PARP) which results in depletion of cellular energy stores (13), stimulate apoptosis, and inhibit mitochondrial complexes I and IV (14).

\section{Endothelial NOS is protective against cerebral ischemia}

In contrast to neuronal NOS, endothelial NOS appears to be protective against cerebral ischemia. When eNOS mutant mice are subjected to the MCAO model of focal ischemia, they develop larger infarcts than do 
wild-type mice (15). Moreover, laser Doppler flowmetry shows that eNOS mutant mice have a more marked blood flow reduction in the ischemic penumbra than do wild-type mice; flow reductions in the core ischemic area are severe in both cases. Exaggerated vascular effects of the ischemia model are also demonstrated independently, using functional CT imaging (16). Following administration of the contrast agent iodohexol, the kinetics of the appearance and disappearance of the contrast can be calculated in each pixel of a CT image. Following MCAO, the total area at risk is the same in the eNOS mutant mice and wild-type mice. However, the eNOS mutant mice show a larger area of no blood flow, reflecting the larger core ischemic area, and have a correspondingly smaller rim of reduced blood flow, reflecting the ischemic penumbra.

In addition to blood flow effects, eNOS may be protective by several additional mechanisms. Endothelial NO production inhibits leukocyte adhesion to the vessel wall and blocks platelet aggregation, both effects that may be relevant to atherosclerotic and thrombotic cerebrovascular disease.

\section{NOS isoforms differentially modulate neurotransmitter release in the brain}

Using microdialysis, the levels of neurotransmitter release can be measured in specific locations in the brain, both at baseline and in response to depolarization and NMDA treatment. Baseline glutamate and gammaaminobutyric acid (GABA) release are unchanged in either nNOS or eNOS knockout mice as compared with wild-type mice (17). Likewise, glutamate and GABA release in response to potassium-stimulated depolarization is also unaffected by gene knockout of either nNOS or eNOS. However, NMDAstimulated glutamate release in the cortex was significantly reduced in the nNOS mutant mice, but unaffected in the striatum and hippocampus. Furthermore, NMDA-stimu- lated GABA release was reduced in all brain regions examined in the eNOS knockout mice (17). These results indicate that nNOS and eNOS isoforms differentially modulated the release of excitatory and inhibitory neurotransmitters in various regions of the brain. These effects may also contribute to the protection against cerebral ischemia seen in the nNOS knockout mice (18), and the worsening of outcome seen in the eNOS knockout mice.

\section{Both nNOS and eNOS may produce NO as a retrograde messenger}

Learning and memory are thought to involve synaptic plasticity, or activity-dependent changes in synaptic strength. Simplified models of these processes include long-term potentiation (LTP) and long-term depression (LTD) in the hippocampus and LTD in the cerebellum. There are several theories about how certain synapses are strengthened or weakened following activity. Some involve post-synaptic changes, such as increase in receptor density or increase in sensitivity to neurotransmission. Others involve presynaptic changes, such as increase in amount of neurotransmitter released. It is likely that at least some component of synaptic plasticity is pre-synaptic. In this case, a retrograde messenger must travel from the post-synaptic neuron to the pre-synaptic neuron to signal that neurotransmission has been received. The identity of the retrograde messenger has been elusive. NO is a strong candidate for retrograde messenger, because it diffuses freely across cell membranes and has a small and precise volume of activity (19-22).

Hippocampal LTP is normal in the neuronal NOS knockout mice and endothelial NOS knockout mice (23). However, one form of LTP located in the stratum radiatum is abnormal in double knockout mice that lack both nNOS and eNOS (24). This indicates that both nNOS and eNOS may generate $\mathrm{NO}$ as a retrograde messenger, and more- 
over, one isoform may substitute for lack of another isoform.

\section{eNOS suppresses formation of neointima following vessel injury}

Endothelial dysfunction describes a condition in which normal physiologic endothelial function is reduced. Functionally, endothelial function may be assayed by flowdependent vasodilation or acetylcholinemediated vasodilation, both of which reflect whether the endothelium produces NO. However, perhaps more important than effects on vascular tone are effects of $\mathrm{NO}$ on cellular proliferation, leukocyte activation, and platelet aggregation, all of which would suppress atherogenesis. However, a causal relationship has not been established between lack of endothelial NO production and increases in cellular proliferation or effects on leukocytes and platelets.

The eNOS mutant mice provided an opportunity to test whether reduction in endothelial NO production would influence the development of neointima following vessel injury. In a cuff model of vessel injury, a nonocclusive polyethylene cuff is placed around the femoral artery (25). The cuff is larger than the vessel outer diameter and does not reduce blood flow in the artery. Fourteen days later, there is reproducible formation of neointima, which can be quantitated by morphometry of sections from the cuffed vessel. The eNOS knockout mice developed far greater neointimal proliferation than did wild-type mice, with intima to media (I/M) volume ratios of 0.70 and 0.42 for males and females, respectively, as compared with $\mathrm{I} / \mathrm{M}$ ratios of 0.30 and 0.18 in wild-type animals (26). Thus, deficiency of eNOS leads to a more pronounced proliferative response to cuff injury, and development of more neointima. As in wild-type animals, there is a gender effect, with more intimal proliferation seen in males than in females. These results indicate that the effect of female gen- der cannot be limited to estrogen-induced increases in the expression of eNOS, since gender differences are noted even in the animals that lack eNOS expression.

\section{eNOS blunts the cardiac systolic contractility response to adrenergic agonists}

The development of small high-fidelity micromanometer-tipped catheters allows the precise measurement of left ventricular pressure tracings in anesthetized, closed-chested animals, despite the high heart rate of 600 beats per minute and the small size of the animal. Left ventricular pressure tracings show similar indices of systolic contractility $(\mathrm{dP} / \mathrm{dt} \max )$ at baseline in wild-type and eNOS knockout mice. However, the dose response of contractility increases to increasing amounts of the $\beta$-adrenergic agonist isoproterenol is steeper in the eNOS mutant mice, indicating that NO normally suppresses the increased contractility response to isoproterenol. The response of wild-type mice treated with the NOS inhibitor L-NA is similar to that of eNOS mutant mice, and L-NA has no additional effects in the eNOS mutants, showing that the eNOS isoform is the only one involved in modulation of cardiac contractility $(27,28)$.

\section{eNOS and ANP both modulate diastolic relaxation of the heart}

The same left ventricular pressure tracings give rise to a time constant of diastolic relaxation, which reflects the stiffness of the left ventricle. Of interest, eNOS mutant mice show normal diastolic relaxation, yet wildtype mice treated with L-NA show increased time constant and stiffer ventricles. Thus, in the case of diastolic relaxation, the eNOS mutant mice behave like wild-type mice, not like wild-type mice treated with NOS inhibitors. These results suggest that despite a normal role for $\mathrm{NO}$ in diastolic relaxation, in 
the absence of eNOS, other processes maintain diastolic relaxation $(27,28)$.

The levels of cGMP in the heart are unchanged by eNOS gene deletion, despite absence of eNOS, an important upstream regulator of guanylyl cyclase. However, the levels of atrial natriuretic peptide (ANP) are upregulated in the heart of the eNOS knockout mice, providing an alternative mechanism for the generation of cGMP. Moreover, administration of exogenous ANP restores ventricular relaxation in L-NA-treated wild-type mice, indicating that ANP is sufficient to restore normal cGMP levels and normal diastolic relaxation. It is unclear whether upregulation of ANP is a physiologic compensation for lack of eNOS, or whether it is a cardiac response to hypertension.

It is important to note that basal systolic cardiac contractility is unchanged in the eNOS mutant mice. However, immediate responses to isoproterenol, which occur on a faster time scale, are exaggerated in the eNOS knockout mice. Thus, the upregulation of ANP, which normalizes basal cGMP levels, does not restore the normal response to adrenergic agonists, since this occurs on a faster moment-to-moment basis.

\section{Conclusion}

Gene knockout of NOS genes provides useful animal models with which to study the roles of individual NOS isoforms in physiologic processes. These animals demonstrate divergent roles of nNOS and eNOS on outcome of cerebral ischemia, highlighting the potential toxicity from nNOS-derived $\mathrm{NO}$ and the vascular protective effects of eNOSderived NO. The knockout mice also indicate how nNOS and eNOS isoforms may substitute for one another in the generation of NO as a retrograde messenger in LTP. Animals deficient in eNOS provide an important link between endothelial dysfunction and neointimal proliferation, which is likely to be an important step in atherogenesis. They also indicate that eNOS and ANP play important roles in modulation of cardiac contractility and relaxation.

\section{References}

1. Moncada S, Palmer RMJ \& Higgs EA (1991). Nitric oxide: physiology, pathophysiology, and pharmacology. Pharmacological Reviews, 43: 109-142.

2. Nathan C \& Xie QW (1994). Nitric oxide synthases: roles, tolls, and controls. Cell, 78: 915-918.

3. Huang PL, Dawson TM, Bredt DS, Snyder SH \& Fishman MC (1993). Targeted disruption of the neuronal nitric oxide synthase gene. Cell, 75: 1273-1286.

4. Brenman J E, Chao DS, Gee SH, McGee AW, Craven SE, Santillano DR, Wu Z, Huang F, Xia H, Peters MF, Froehner SC \& Bredt DS (1996). Interaction of nitric oxide synthase with the post synaptic density protein PSD-95 and alphalsyntrophin mediated by PDZ domain. Cell, 84: 757-767.

5. Mashimo $H$, He XD, Huang PL, Fishman MC \& Goyal RK (1996). Neuronal constitutive nitric oxide synthase is involved in murine enteric inhibitory neurotransmission. J ournal of Clinical Investigation, 98:
8-13.

6. Huang $\mathrm{PL}$, Huang $\mathrm{Z}$, Mashimo $\mathrm{H}$, Bloch KD, Moskowitz MA, Bevan J A \& Fishman MC (1995). Hypertension in mice lacking the gene for endothelial nitric oxide synthase. Nature, 377: 239-242.

7. Malinski T, Bailey F, Zhang ZG \& Chopp M (1993). Nitric oxide measured by a porphyrinic microsensor in rat brain after transient middle cerebral artery occlusion. J ournal of Cerebral Blood Flow and Metabolism, 13: 355-358.

8. Huang Z, Huang PL, Panahian N, Dalkara T, Fishman MC \& Moskowitz MA (1994). Effects of cerebral ischemia in mice deficient in neuronal nitric oxide synthase. Science, 265: 1883-1885.

9. Hara $\mathrm{H}$, Huang $\mathrm{PL}$, Panahian $\mathrm{N}$, Fishman MC \& Moskowitz MA (1996). Reduced brain edema and infarction volume in mice lacking the neuronal isoform of nitric oxide synthase after transient MCA occlusion. J oumal of Cerebral Blood Flow and Metabolism, 16: 605-611.
10. Panahian N, Yoshida T, Huang PL, HedleyWhyte ET, Fishman M \& Moskowitz MA (1996). Attenuated hippocampal damage after global cerebral ischemia in knockout mice deficient in neuronal nitric oxide synthase. Neuroscience, 72: 343-354.

11. Koppenol WH, Moreno JJ, Pryor WA, Ischiropoulos H \& Beckman JS (1992). Peroxynitrite, a cloaked oxidant formed by nitric oxide and superoxide. Chemical Research in Toxicology, 5: 834-842.

12. Ayata C, Ayata G, Hara H, Matthews RT, Beal MF, Ferrante RJ , Endres M, Kim A, Christie RH, Waeber C, Huang PL, Hyman BT \& M oskowitz MA (1997). Mechanisms of reduced striatal NMDA excitotoxicity in type I nitric oxide synthase knock-out mice. J ournal of Neuroscience, 17: 69086917.

13. Endres $M$, Scott $G$, Namura $S$, Salzman AL, Huang PL, Moskowitz MA \& Szabo C (1998). Role of peroxynitrite and neuronal nitric oxide synthase in the activation of poly(ADP-ribose) synthetase in a murine 
model of cerebral ischemia-reperfusion. Neuroscience Letters, 248: 41-44.

14. Clementi E, Brown GC, Feelisch $M$ \& Moncada S (1998). Persistent inhibition of cell respiration by nitric oxide: crucial role of S-nitrosylation of mitochondrial complex I and protective action of glutathione. Proceedings of the National Academy of Sciences, USA, 95: 7631-7636.

15. Huang Z, Huang PL, MaJ , Meng W, Ayata C, Fishman MC \& Moskowitz MA (1996). Enlarged infarcts in endothelial nitric oxide synthase knockout mice are attenuated by nitro-L-arginine. J ournal of Cerebral Blood Flow and Metabolism, 16: 981987.

16. Lo EH, Hara H, Rogowska J, Trocha M, Pierce AR, Huang PL, Fishman MC, Wolf GL \& Moskowitz MA (1996). Temporal correlation mapping analysis of the hemodynamic penumbra in mutant mice deficient in endothelial nitric oxide synthase gene expression. Stroke, 27: 1381-1385.

17. Kano $T$, Shimizu-Sasamata $M$, Huang $P L$, Moskowitz MA \& Lo EH (1998). Effects of nitric oxide synthase gene knockout on neurotransmitter release in vivo. Neuroscience, 86: 695-699.

18. Shimizu-Sasamata $M$, Bosque-Hamilton $P$, Huang PL, Moskowitz MA \& Lo EH
(1998). Attenuated neurotransmitter release and spreading depression-like depolarizations after focal ischemia in mutant mice with disrupted type I nitric oxide synthase gene. J ournal of Neuroscience, 18: 9564-9571.

19. Bohme GA, Bon C, Stutzman J M, Doble A \& Blanchard J C (1991). Possible involvement of nitric oxide in long-term potentiation. European J ournal of Pharmacology, 199: 379-381.

20. Haley JE, Wilcox GL \& Chapman PF (1992). The role of nitric oxide in hippocampal long-term potentiation. Neuron, 8: 211-216.

21. O'Dell TJ, Hawkins RD, Kandel ER \& Arancio O (1991). Tests of the roles of two diffusable substances in long term potentiation: evidence for nitric oxide as a possible early retrograde messenger. Proceedings of the National Academy of Sciences, USA, 88: 11285-11289.

22. Schuman EM \& Madison DV (1991). A requirement for the intercellular messenger nitric oxide in long-term potentiation. Science, 254: 1503-1506.

23. O'Dell TJ, Huang PL, Dawson TM, Dinerman J L, Snyder SH, Kandel ER \& Fishman MC (1994). Endothelial NOS and the blockade of LTP by NOS inhibitors in mice lacking neuronal NOS. Science, 265 : 542-546.

24. Son $\mathrm{H}$, Hawkins RD, Martin $\mathrm{K}$, Kiebler $\mathrm{M}$, Huang PL, Fishman MC \& Kandel ER (1996). Long-term potentiation is reduced in mice that are doubly mutant in endothelial and neuronal nitric oxide synthase. Cell, 87: 1015-1023.

25. Booth RFG, Martin J F, Honey AC, Hassall DG, Beesley JE \& Moncada S (1989). Rapid development of atherosclerotic lesions in the rabbit carotid artery induced by perivascular manipulation. Atherosclerosis, 76: 257-268.

26. Moroi $M$, Zhang $L$, Yasuda $T$, Virmani $R$, Gold HK, Fishman MC \& Huang PL (1998). Interaction of genetic deficiency of endothelial nitric oxide, gender, and pregnancy in vascular response to injury in mice. J ournal of Clinical Investigation, 101: 1225-1232.

27. Huang PL \& Lo EH (1998). Genetic analysis of NOS isoforms using nNOS and eNOS knockout animals. Progress in Brain Research, 118: 13-25.

28. Huang PL (1998). Disruption of the endothelial nitric oxide synthase gene: effect on vascular response to injury. American J ournal of Cardiology, 82: 57S-59S. 